\title{
UM ESTUDO DO CONJUNTO MULTILÍNGUE INTERPESSOAL PORTUGUÊS BRASILEIRO/INGLÊS SUBSIDIADO PELOS ESTUDOS DA TRADUÇÃO E PELA LINGUÍSTICA SISTÊMICO FUNCIONAL
}

\author{
Giacomo Patrocinio Figueredo* \\ Universidade Federal de Ouro Preto
}

\begin{abstract}
Resumo: Este artigo investiga as relações de tradução dos sistemas interpessoais da gramática do português brasileiro e do inglês e seu comportamento no ambiente multilíngue, objetivando estabelecer as relações de equivalência tradutória do estrato gramatical no ambiente multilíngue para modelar recursos gramaticais. Procura-se aqui contribuir com as abordagens sistêmicas da tradução, na sua vertente da produção multilíngue, com o desenvolvimento de um modelo do ambiente multilíngue, a partir das relações de equivalência, correspondência e mudança. Para tanto, toma-se, como hipótese de trabalho, que o ambiente multilíngue pode ser modelado como o conjunto universo dos recursos gramaticais interpessoais da oração, empregando as relações de equivalência em tradução, correspondência e mudança como ferramentas dessa investigação. Torna-se assim possível descrever os subconjuntos relativos tanto às línguas quanto aos recursos comuns a elas - aqui denominados multilíngues. A partir daí, o comportamento gramatical do contato linguístico, por sua vez, passa a se constituir como uma variação do uso da língua. Os resultados mostram como empregar as relações de equivalência, correspondência e mudança em tradução como ferramentas metodológicas para modelar um ambiente multilíngue. Além disso, indicam como o conceito 'multilíngue' refere-se
\end{abstract}

\footnotetext{
* Possui mestrado e doutorado em Estudos Linguísticos pela Universidade Federal de Minas Gerais (2011). É professor da Universidade Federal de Ouro Preto. Ouro Preto, Minas Gerais, Brasil. E-mail giacomojakob@yahoo.ca
} 
tanto à pervasão de sistemas no ambiente multilíngue quanto ao comportamento gramatical das línguas.

Palavras-chave: Abordagens sistêmicas da tradução. Estudos multilíngues. Modelagem de significado interpessoal. Topologia linguística.

\title{
A MULTILINGUAL SET EXPLORATION OF INTERPERSONAL GRAMMAR IN BRAZILIAN PORTUGUESE/ENGLISH UNDER A TRANSLATION STUDIES AND SYSTEMIC FUNCTIONAL THEORY FRAMEWORK
}

\begin{abstract}
The paper explores translation in interpersonal grammar systems of Brazilian Portuguese and English, identifying its behavior in a multilingual environment. It aims at deploying translation equivalence, correspondence and shift at the grammar stratum in the multilingual environment to model interpersonal clause grammar. The multilingual environment is as a result modeled as the power set of resources in both languages and translation equivalence, formal correspondence and shifts are taken as tools for such modeling. Subsets of language-specific resources and their intersections - i.e., multilingual - between them can then be described. Language contact behavior is described through translation equivalence and shift, since it is a variation of language deployment. Through such modeling this paper shows how equivalence, correspondence and shift can be used as tools to model a multilingual environment. Moreover, it suggests the notion of 'multilingual' may refer both to the pervasive nature of grammar systems and grammar behavior of languages when in contact.
\end{abstract}

Keywords: Systemic approaches to translation. Multilingual studies. Interpersonal Meaning modeling. Language topology.

O presente artigo está localizado na complementaridade entre as Abordagens Sistêmicas da Tradução (PAGANO e VASCONCELLOS, 2005) e os Estudos Multilíngues (MATTHIESSEN et al., 2008), e delimita como objeto a produção de significado gramatical no ambiente multilíngue.

Se por um lado os Estudos da Tradução possuem como objeto de estudo os fenômenos relacionados à tradução, os Estudos 
Multilíngues (MATTHIESSEN et al., 2008), por outro, procuram estabelecer uma base comum para as áreas de investigação dos fenômenos que envolvem mais de uma língua, tais como o estudo da tradução e a linguística contrastiva.

Para investigar seu objeto, os Estudos da Tradução lançam mão de diferentes abordagens e metodologias, como por exemplo aquelas que guiam a presente pesquisa - a análise linguística e a variação de registros (TEICH, 2003). Já o campo disciplinar dos Estudos Multilíngues visa estabelecer relações complementares entre as diversas formas de se abordar os fenômenos do contato linguístico (STEINER e YALLOP, 2001).

A relação entre os Estudos da Tradução e os Estudos Multilíngues ganha motivação em pesquisas anteriores (TEICH, 1999; MATTHIESSEN et al., 2008), as quais mostram que a investigação dos fenômenos tradutórios revela uma maior amplitude do potencial das línguas envolvidas quando estabelece relações com outros fenômenos da produção multilíngue. Os Estudos da Tradução e os Estudos Multilíngues se complementam, portanto, no sentido de que o fenômeno tradutório é visto pelos últimos como um tipo especializado de produção de significado linguístico.

Devido ao caráter de campo disciplinar emergente, os Estudos Multilíngues têm como desafio desenvolver um modelo analítico que consiga investigar a produção multilíngue a partir de categorias descritivas tão diversas quanto as próprias línguas envolvidas. No entanto, dentro dos Estudos Multilíngues não existe ainda uma metodologia consolidada que possa determinar os critérios de análise do modelo. Nesse ponto, os Estudos da Tradução podem oferecer uma base comum de investigação, por se verificarem relações de tradução entre quaisquer línguas.

Este artigo, procurando contribuir para a determinação de critérios de análise do modelo, emprega o estudo da tradução como ferramenta metodológica para a investigação do contraste linguístico no ambiente multilíngue. Para tanto, tem como objetivo principal estabelecer as relações de equivalência tradutória (CATFORD, 1965; MATTHIESSEN, 2001) do estrato gramatical no ambiente 
multilíngue segundo o arcabouço dos Estudos da Tradução e balizados pela Linguística Sistêmico Funcional (cf. HALLIDAY e MATTHIESSEN, 2013) - especificamente, a pesquisa se concentra nos sistemas gramaticais interpessoais, a saber, MODO, MODALIDADE, POLARIDADE e AVALIAÇÃO MODAL. Em seguida, emprega as relações de equivalência como método para o estudo de outros tipos de relação entre línguas - mais especificamente ao contraste (ou relações descritivas formais) dos sistemas gramaticais interpessoais em português brasileiro (doravante, PB) e inglês (FIGUEREDO 2011; HALLIDAY e MATTHIESSEN, 2013).

$\mathrm{O}$ restante do artigo se organiza da seguinte forma. A próxima seção apresenta o modo pelo qual é feita a modelagem do ambiente multilíngue, a partir das relações de tradução. Em seguida, é apresentada a metodologia. A seção de análise mostra como as relações de equivalência em tradução e correspondência podem ser utilizadas para a comparação entre as línguas relativamente a outros fenômenos do contato linguístico e, subsequentemente, a análise da equivalência e da mudança apresenta as contribuições deste trabalho para o estudo da tradução e do contraste linguístico a partir do comportamento gramatical multilíngue. Por fim, as conclusões retomam as possíveis implicações do trabalho para os campos disciplinares das Abordagens Sistêmicas da Tradução e dos Estudos Multilíngues.

\section{As relações de tradução na modelagem de ambientes multilíngues}

Halliday (2002) aponta, talvez, a principal questão para a investigação da tradução e do contraste entre línguas: a descrição das línguas pode ser (a) generalizada ou (b) comparável? Esse autor (2002, p. 21) afirma:

A descrição de uma determinada língua (...) pode partir da organização formal da própria língua, (...) de outras línguas, ou ainda de critérios não-formais de organização (...). 
Cumpre ressaltar que não existem categorias linguísticas de organização formal universais. Hipoteticamente, poderia haver categorias que, formalmente, são comuns a todas as línguas, mas na realidade isso não é possível. Quanto aos critérios não-formais, esses só caberiam na descrição se forem considerados, a priori, como universais. ${ }^{1}$

Conforme Halliday, a resposta da questão é sempre arbitrária, quer pela inexistência de categorias universais, quer pela inadequação dos critérios não-formais. O estudo multilíngue - incluindo-se a tradução e o contraste - portanto, parte sempre de uma hipótese que, apesar de não poder ser provada por experimento, segue metodologias que levam a resultados replicáveis, capazes de explicar as relações entre as línguas.

Diante dessa consideração, a modelagem de ambientes multilíngues aqui apresentada parte da seguinte hipótese de trabalho: um ambiente multilíngue é o conjunto de todos os recursos de todas as linguas que constituem esse ambiente.

Um ambiente multilíngue (aqui denominado $\mathrm{M}^{2}$ ) se define como o conjunto universo que contém todos os recursos sistêmicos das línguas sob investigação para, especificamente, esse ambiente. Nessas condições, as línguas se tornam subconjuntos do ambiente multilíngue. Por conseguinte, $\mathrm{M}$ se relaciona a uma determinada língua (aqui denominada L) que dele forma parte:

$$
\mathrm{P}(\mathrm{M})=\{\mathrm{L} \mid \mathrm{L} \subset \mathrm{M}\}
$$

Uma instância de $\mathrm{M}$ que se limita ao estrato lexicogramatical é o conjunto universo para todos os recursos lexicogramaticais das línguas $\mathrm{P}(\mathrm{M})$. Gramaticalmente, uma língua L é definida como o conjunto de recursos lexicogramaticais (aqui denominado R) que constituem as opções funcionais (aqui denominadas F) dessa mesma língua:

$$
\mathrm{L}=\{\mathrm{R} \in \mathrm{M} \mid \mathrm{R}=\mathrm{F}\}
$$


Devido ao fato de os recursos lexicogramaticais constituírem as línguas, uma determinada língua $\mathrm{L}$ também se relaciona a $\mathrm{M}$ por meio de R:

$$
\mathrm{L} \subset \mathrm{M} \Leftrightarrow(\forall \mathrm{R})(\mathrm{R} \in \mathrm{L} \Rightarrow \mathrm{R} \in \mathrm{M})
$$

Quando mais de uma língua está presente em M (por exemplo, línguas $\mathrm{L}_{1}$ e $\mathrm{L}_{2}$ ), a união delas também se relaciona tanto a $\mathrm{M}$ quanto a R:

$$
\mathrm{M}=\left\{\mathrm{L}_{1} \cup \mathrm{L}_{2}\right\}=\left\{\mathrm{R} \mid \mathrm{R} \in \mathrm{L}_{1} \vee \mathrm{R} \in \mathrm{L}_{2}\right\}
$$

Isto implica em que os recursos de $\mathrm{R}$ sejam recursos "supra-línguas" (i.e., multilíngues) quando tomados como elementos de um ambiente multilíngue; se $\mathrm{R} \in \mathrm{L}_{1}$ ou $\mathrm{R} \in \mathrm{L}_{2}$, então $\mathrm{R} \in \mathrm{M}$. Com isso, se dois recursos de duas línguas possuem o mesmo valeur (i.e., opções do mesmo sistema, no mesmo nível de delicadeza) são modelados como o mesmo recurso no ambiente multilíngue, e as manifestações nas línguas são modeladas como homólogas.

Os elementos de $\mathrm{R}$ também se relacionam a um ambiente multilíngue porque $\mathrm{M}$ pode ser modelado como o universo para subconjuntos de (i) um determinado número de línguas; (ii) uma língua L (ou línguas $\mathrm{L}_{1}, \mathrm{~L}_{2}$, etc.) e um conjunto de recursos não-determinados (aqui denominados $\mathrm{Rx}$ ), cuja pertinência às línguas $\mathrm{L}_{1}$ ou $\mathrm{L}_{2}$, etc. deverá ser determinada durante o curso da pesquisa; (iii) um conjunto de recursos indeterminados (Ry) gerados pelo contato entre as línguas, que não pertencem a nenhuma das línguas presentes em M e, da mesma forma, deverão ser determinados pela pesquisa. Tais relações podem ser sistematizadas da seguinte maneira:

$$
\begin{aligned}
& \text { (i) } \mathrm{M}=\mathrm{L}_{1} \cup \mathrm{L}_{2} \\
& \text { (ii) } \mathrm{Rx} \in \mathrm{L}_{1} \vee \mathrm{Rx} \Leftrightarrow \mathrm{L}_{2} \forall(\subset \mathrm{Rx})(\mathrm{Rx} \square \mathrm{R}) \\
& \text { (iii) } \mathrm{Ry}=\mathrm{M}-\mathrm{L}_{1} \cup \mathrm{L}_{2} \neq \varnothing
\end{aligned}
$$

Quando se examina $\mathrm{M}$ em relação a uma língua específica $\mathrm{L}$, então $\mathrm{M}$ é o universo de $\mathrm{L}$ e do conjunto de todos os recursos que 
não pertencem a $\mathrm{L}$ - ou o complementar da língua $\mathrm{L}$ (aqui denominado $\left.\mathrm{L}^{\mathrm{C}}\right)$. Se mais de uma língua estiver presente em $\mathrm{M}\left(\mathrm{L}_{1}, \mathrm{~L}_{2}\right.$, etc.), aplica-se a mesma complementaridade:

$$
\begin{aligned}
\mathrm{L}^{\mathrm{C}} & =\mathrm{M}-\mathrm{L} \\
\left(\mathrm{L}_{1} \cup \mathrm{L}_{2}\right)^{\mathrm{C}} & =\mathrm{M}-\left(\mathrm{L}_{1} \cup \mathrm{L}_{2}\right)
\end{aligned}
$$

A consequência disso, então, é que a descrição exaustiva dos recursos de todas as línguas presentes em um ambiente multilíngue, juntamente com seus complementares é igual ao total de recursos presentes nesse ambiente multilíngue:

$$
\mathrm{L}_{1} \cup \mathrm{L}_{1}{ }^{\mathrm{C}}=\mathrm{L}_{2} \cup \mathrm{L}_{2}{ }^{\mathrm{C}} \cup \mathrm{L}_{1} \cup\left(\mathrm{M}-\mathrm{L}_{1}\right)=\mathrm{L}_{2} \cup\left(\mathrm{M}-\mathrm{L}_{2}\right) \Rightarrow \mathrm{M}=\mathrm{M}
$$

Isso significa que todas as línguas que são subconjuntos de $\mathbf{M}$ pertencem à mesma classe. Por esse motivo, não é necessário classificar as línguas para além de suas relações com M, R, ou seus complementares. Contudo, ressalta-se que caso alguma distinção entre as línguas seja feita, ela se deve a fatores externos ao modelo, e dependerá dos interesses diversos dos campos disciplinares, tais como línguas fonte e meta nos Estudos da Tradução, ou línguas materna e estrangeira na Linguística Aplicada ao Ensino de LE. Ressalta-se que, por outro lado, no ambiente multilíngue, uma língua pode ser "derivada" a partir de qualquer outra língua, na relação com $\mathrm{M}$.

$$
\begin{gathered}
\mathrm{L}_{1} \cup \mathrm{L}_{1}{ }^{\mathrm{C}}=\mathrm{L}_{2} \cup \underset{2}{\mathrm{~L}_{2}{ }^{\mathrm{C}} \Rightarrow} \mathrm{L}_{1}=\left(\mathrm{L}_{1} \cup \mathrm{L}_{1}{ }^{\mathrm{C}}-\mathrm{L}_{2}{ }^{\mathrm{C}}\right) \cup \mathrm{L}_{2}{ }^{\mathrm{C}} \mathrm{L}_{1}{ }^{\mathrm{C}} \Rightarrow \mathrm{L}_{1}=\mathrm{L}_{1} \Rightarrow \mathrm{L}_{2}= \\
\left(\mathrm{L}_{2} \cup \mathrm{L}_{2}{ }^{\mathrm{C}}-\mathrm{L}_{1}{ }^{\mathrm{C}}\right) \cup \mathrm{L}_{1}{ }^{\mathrm{C}}-\mathrm{L}_{2}{ }^{\mathrm{C}} \Rightarrow \mathrm{L}_{2}=\mathrm{L}_{2}
\end{gathered}
$$

Uma vez que todas as línguas em $\mathrm{M}$ são classificadas como subconjuntos, as relações entre elas são (para um universo $\mathrm{M}$, que tem apenas as línguas $\mathrm{L}_{1}$ e $\mathrm{L}_{2}$ como subconjuntos).

(1) Disjunção: quando as línguas $\mathrm{L}_{1}$ e $\mathrm{L}_{2}$ não possuem recursos comuns, e com isso, são completamente diferentes. 


$$
\mathrm{L}_{1} \cap \mathrm{L}_{2}=\varnothing
$$

(2) Anti-simetria: $\mathrm{L}_{1}$ e $\mathrm{L}_{2}$ compartilham todos os recursos e, com isso, são iguais.

$$
\mathrm{L}_{1}=\mathrm{L}_{2} \Rightarrow \mathrm{L}_{1} \cup \mathrm{L}_{2}{ }^{\mathrm{C}}-\mathrm{L}_{1}{ }^{\mathrm{C}}=\mathrm{L}_{1} \cup \mathrm{L}_{1}{ }^{\mathrm{C}}-\mathrm{L}_{1}{ }^{\mathrm{C}} \Rightarrow \mathrm{L}_{1}=\mathrm{L}_{1}
$$

(3) Interseção: se as línguas $\mathrm{L}_{1}$ e $\mathrm{L}_{2}$ compartilham um conjunto comum de recursos (denominado $\mathrm{X}$ ), então $\mathrm{L}_{1} \cap \mathrm{L}_{2}=\mathrm{X}$. Ressaltam-se aqui duas consequências importantes desta relação. Primeiramente, do ponto de vista da língua $\mathrm{L}_{1}$, a língua $\mathrm{L}_{2}$ é igual aos recursos que são compartilhados:

$$
\mathrm{L}_{1}-\mathrm{L}_{2}=\mathrm{L}_{1}-\mathrm{X} \Rightarrow \mathrm{L}_{1}-\mathrm{X}-\mathrm{L}_{1} \cup \mathrm{L}_{2}=0 \Rightarrow \mathrm{L}_{2}=\mathrm{X}
$$

Em segundo lugar, torna-se possível estabelecer recursos ortológicos entre as línguas. Para qualquer $\mathrm{M}=\mathrm{L}_{1} \cup \mathrm{L}_{2}$, o complementar da língua $\mathrm{L}_{1}$ é a língua $\mathrm{L}_{2}$, com exceção dos recursos compartilhados X:

$$
\begin{aligned}
& \mathrm{L}_{1}{ }^{\mathrm{C}}=\mathrm{L}_{2}-\mathrm{X} \Rightarrow \mathrm{X}=\mathrm{L}_{2}-\mathrm{L}_{1}{ }^{\mathrm{C}} \\
& \mathrm{L}_{2}{ }^{\mathrm{C}}=\mathrm{L}_{1}-\mathrm{X} \Rightarrow \mathrm{X}=\mathrm{L}_{1}-\mathrm{L}_{2}{ }^{\mathrm{C}}
\end{aligned}
$$

Essa propriedade é importante para o modelo, pois a extensão dos recursos compartilhados entre as línguas (i.e., X) é tão importante quanto a própria descrição das línguas - e dos recursos que as constituem. Essa propriedade mostra que existe uma linha (que nem sempre é absolutamente clara) que separa os recursos de $\mathrm{L}_{1}$ (i.e., $\mathrm{L}_{1}-\mathrm{L}_{2}$ ) daqueles que são compartilhados com $\mathrm{L}_{2}$ (i.e., $\mathrm{L}_{1}-\mathrm{L}_{2}{ }^{\mathrm{C}}$ ). $\mathrm{Na}$ região de fora da linha estão os recursos multilíngues para as línguas individualmente. Na parte de dentro da linha, estão os recursos compartilhados pelas línguas (i.e., $\mathrm{X}=\mathrm{L}_{1}-\mathrm{L}_{2}{ }^{\mathrm{C}}=\mathrm{L}_{2}-\mathrm{L}_{1}{ }^{\mathrm{C}}$ ) (Figura 1). 


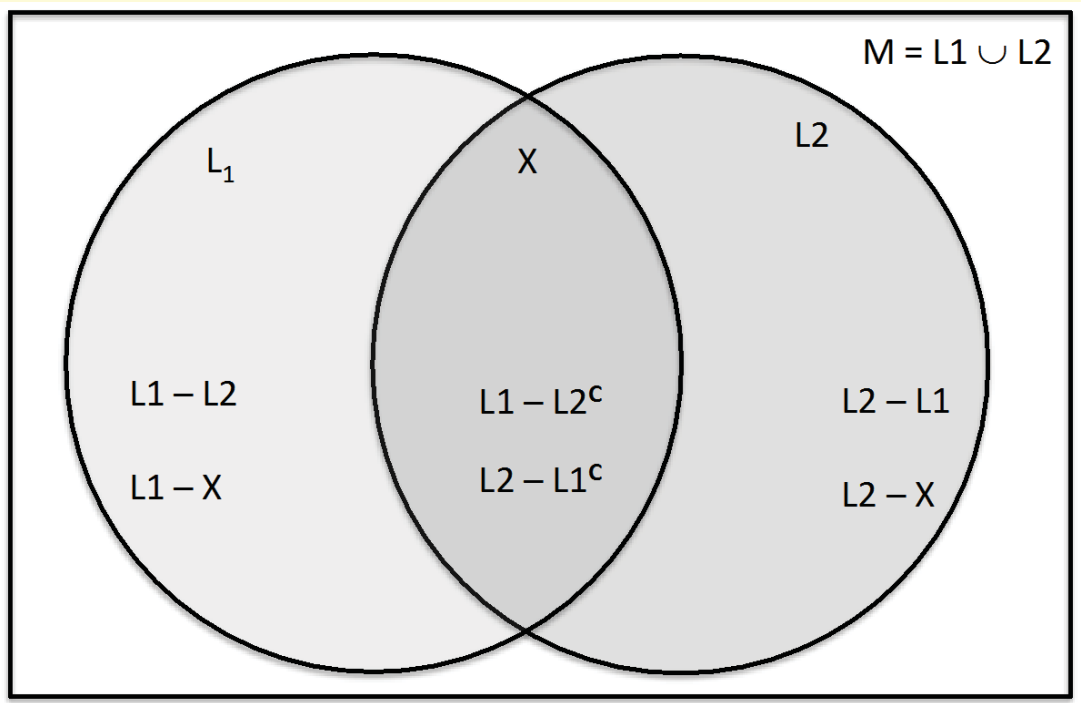

FIGURA 1 - Interseção entre $\mathrm{L}_{1}$ e $\mathrm{L}_{2}$.

Diante dessas considerações, sistematiza-se assim a modelagem:

a. Na perspectiva dos Estudos Multilíngues (MATTHIESSEN et al., 2008), o ambiente multilíngue se caracteriza como o espaço constituído pela produção de significado linguístico a partir das relações paradigmáticas entre funções (MARTIN, 2013).

b. O ambiente multilíngue é um modelo da produção de significado. Assim, o sistema linguístico é supra-línguas (multilíngue) e as línguas são subconjuntos do ambiente multilíngue. Os recursos das línguas também pertencem ao ambiente, e a separação entre as línguas se dá no limite entre sua Interseção e Disjunção.

c. Todas as línguas pertencem à mesma classe, por isso são classificadas da mesma forma em um ambiente multilíngue, uma vez que todas mantêm relação de subconjunto com aquele. 


\section{Metodologia}

Tomando os sistemas da gramática interpessoal (MODO, MODALIDADE, POLARIDADE e AVALIAÇÃO MODAL) em PB [aqui denominado $\mathrm{L}_{1}$ ] e inglês [aqui denominado $\mathrm{L}_{2}$ ] como o foco de investigação, empregou-se a tradução como ferramenta metodológica na investigação do contraste linguístico, utilizando as relações de equivalência em tradução, correspondência formal e mudança na tradução (CATFORD, 1965). A metodologia deste artigo se dividiu em cinco etapas.

(1) Compilação. O corpus foi compilado observando-se que os textos (a) estivessem em relação de tradução e fossem representativos das duas direções; (b) demandassem trabalho gramatical dos sistemas interpessoais. Com isso, foram selecionados diálogos falados de negociação aberta, uma vez que, prototipicamente, exploram o potencial interpessoal da gramática (cf. MARTIN, 1992). Por fim, (c) foram compilados textos com o uso controlado da língua - em oposição a espontâneo - pois tende a se restringir às funções mais frequentes dos sistemas. Desta maneira, foi utilizada como base para a compilação a tipologia da língua no contexto de cultura. Essa tipologia é definida segundo cinco variáveis, as quais refletem a organização do modelo metafuncional da língua (MATTHIESSEN et al. 2008; HALLIDAY e MATTHIESSEN, 2013):

Especialização, segundo a qual leva-se em conta o fato de o texto ser produzido ou não pelo conhecimento técnico de uma determinada área (especializado/não-especializado). Papel da língua na situação, na qual o texto é visto como fundamental para a situação sócio-cultural, ou apenas como facilitador da ocorrência da situação (constitutivo/auxiliar). Modo de produção, em que se observa a forma pela qual o texto foi originalmente produzido pelo falante (escrito/falado). Modo de interação, considerando-se o tipo de relação estabelecida textualmente entre os interlocutores, pressupondo a resposta ou não do ouvinte (monólogo/diálogo). Processo sócio-semiótico, que leva em consideração a forma pela qual os textos estão dispersos no contexto de cultura, dividindo-se, confor- 
me os processos sócio-semióticos, em textos para explorar, explicar, relatar, recriar, compartilhar, fazer, recomendar ou habilitar.

Foram selecionados textos não-especializado/constitutivo/falado/diálogo/recriar, que envolvem negociação. Foram selecionadas as transcrições de um trecho do filme Os Normais (trecho 01:11:48 --> 01:13:04) e do seriado The Middle (Temporada 1, episódio 4, trecho 00:12:52 --> 00:13:53), com suas respectivas legendas traduzidas. Em seguida, os textos foram agrupados nos tipos monolíngue comparável - ou seja, um subcorpus composto por textos originais/traduzidos em uma só língua; e paralelo bilíngue - ou o subcorpus dos textos originais e traduções (cf. KENNING, 2010) (Tabela 1).

\begin{tabular}{l|l|l}
\hline Subcorpora & Descrição & Tokens \\
\hline 1 Normais & Transcrição do áudio PB & 290 \\
\hline 2 Middle & Transcrição do áudio inglês & 220 \\
\hline 3 Normais & Legenda traduzida inglês & 179 \\
\hline 4 Middle & Legenda traduzida PB & 197 \\
\hline 5 paralelo bilíngue & Paralelo AB (Normais e Normais-Legenda & 460 \\
\hline 6 paralelo bilíngue & Paralelo BA (Middle e Middle-Legenda) & 417 \\
\hline $\begin{array}{l}7 \text { monolíngue } \\
\text { comparável }\end{array}$ & A-PB (Normais e Middle-Legenda) & 487 \\
\hline $\begin{array}{l}8 \text { monolíngue } \\
\text { comparável }\end{array}$ & B- Inglês (Middle e Normais-Legenda) & 339 \\
\hline
\end{tabular}

TABELA 1 - Corpus da pesquisa.

(2) Nível de análise. Tendo a oração como unidade de análise, foram selecionados os sistemas de MODO, MODALIDADE, POLARIDADE e AVALIAÇÃO MODAL. Para cada sistema, determinou-se a delicadeza - ou o detalhamento e especificidade das categorias gramaticais (GLEASON, 1965) - no terceiro nível, portanto no terceiro subsistema. Para a escolha das funções, tomou-se como base as descrições existentes para as línguas inglês e PB (HALLIDAY e MATTHIESSEN, 2013; FIGUEREDO 2011, respectivamente). Levou-se em conta o fato de que, potencialmen- 
te, as funções poderiam estar presentes em uma ou outra língua, ou $\mathrm{M}=\left\{\mathrm{R} \mid \mathrm{R} \in \mathrm{L}_{1} \vee \mathrm{R} \in \mathrm{L}_{2}\right\}$ (Quadro 1).

QUADRO 1 - Nível de delicadeza da análise e categorias funcionais.

\begin{tabular}{|c|c|c|}
\hline \multicolumn{3}{|c|}{$\mathrm{M}=\left\{\mathrm{R} \mid \mathrm{R} \in \mathrm{L}_{1} \vee \mathrm{R} \in \mathrm{L}_{2}\right\}$} \\
\hline Nível 1 & Nível 2 & Nível 3 \\
\hline \multirow[t]{4}{*}{ modo } & \multirow[t]{2}{*}{ indicativo } & declarativo \\
\hline & & interrogativo \\
\hline & \multirow[t]{2}{*}{ imperativo } & jussivo \\
\hline & & sugestivo \\
\hline \multirow[t]{7}{*}{ modalidade } & \multirow[t]{2}{*}{ modalização } & frequência \\
\hline & & probabilidade \\
\hline & \multirow[t]{2}{*}{ modulação } & obrigação \\
\hline & & inclinação \\
\hline & \multirow[t]{2}{*}{ grau } & baixo \\
\hline & & médio \\
\hline & & alto \\
\hline \multirow[t]{2}{*}{ polaridade } & positiva & \\
\hline & negativa & \\
\hline \multirow[t]{4}{*}{ avaliação modal } & \multirow[t]{2}{*}{ persuasão } & obediência \\
\hline & & resposta \\
\hline & \multirow[t]{2}{*}{ verificação } & prosódia \\
\hline & & papel-falante \\
\hline
\end{tabular}

(3) Descrição. Os recursos da análise foram dispostos como os sistemas particularizados do corpus. Uma vez que, em geral, a descrição é pensada para a língua como um todo, o corpus no qual ela se baseia é representativo de toda a língua. No caso da presente pesquisa, o ambiente multilíngue é a descrição que se limita ao corpus. Foram recuperados do corpus os recursos que se tornam elementos do ambiente multilíngue, $\mathrm{M}=\left\{\mathrm{L}_{1} \cup \mathrm{L}_{2}\right\}=\left\{\mathrm{R} \mid \mathrm{R} \in \mathrm{L}_{1} \vee \mathrm{R} \in \mathrm{L}_{2}\right\}$. 
(4) Estabelecimento dos recursos gramaticais (elementos $R$ ) no ambiente multilíngue (M). Analisadas as funções gramaticais e assim encontrados os recursos das línguas, elas foram correlacionadas com o ambiente multilíngue em duas etapas.

(4.1) Emprego das relações de equivalência e correspondência para a definição dos subconjuntos. Quando duas línguas compõem o ambiente multilíngue, $\mathrm{M}$ é composto por três subconjuntos relevantes: $\mathrm{L}_{1}, \mathrm{~L}_{2}$, e $\mathrm{X}$ (determinado pela confluência entre as relações de Equivalência e Correspondência Formal) (Quadro 2).

QUADRO 2 - Determinação de $\mathrm{L}_{1} \cap \mathrm{L}_{2}=\mathrm{X}$ por Equivalência e Correspondência.

\begin{tabular}{|c|c|c|}
\hline EQUIVALÊNCIA & CORRESPONDÊNCIA & TIPO DE RELAÇÃO \\
\hline$\checkmark$ & $\checkmark$ & $\mathrm{L}_{1} \cap \mathrm{L}_{2}=\mathrm{X}$ \\
\hline$\checkmark$ & $\mathrm{x}$ & $-\cdots--$ \\
\hline $\mathrm{x}$ & $\checkmark$ & $-\cdots$ \\
\hline $\mathrm{x}$ & $\mathrm{x}$ & $\mathrm{L}_{1} \cap \mathrm{L}_{2}=\varnothing$ \\
\hline
\end{tabular}

(4.2) Modelagem do ambiente multilíngue. O estudo de um ambiente multilíngue envolve tanto a descrição dos subconjuntos $\mathrm{L}_{1}$, $\mathrm{L}_{2}$ e $\mathrm{X}$, quanto dos limites que os separam. Tendo como parâmetros as relações Disjunção, Anti-simetria e Interseção, aplicaram-se os recursos de $\mathrm{L}_{1 \ldots .}$ em $\mathrm{L}_{2 \ldots 1} \mathrm{e}$, em seguida, registrou-se sua funcionalidade na determinação dos subconjuntos. Isto possibilitou a comparação os recursos de $\mathrm{L}_{1}$ e $\mathrm{L}_{2}$ para determinar os limites que separam os subconjuntos.

Uma vez descritos os recursos, com a metodologia apresentada até aqui, tornou-se possível modelar o ambiente multilíngue. Contudo, para se explicar o comportamento dos recursos como variação especifica em M, cabe ainda um outro passo.

(5) Estabelecimento do comportamento gramatical multilíngue. Uma vez que $X$ é determinado pela Interseção de $\mathrm{L}_{1}, \mathrm{~L}_{2}$ em $\mathrm{M}, \mathrm{X}$ não captura a Interseção entre PB e inglês. Tendo em vista que o 
comportamento de $\mathrm{L}_{1}, \mathrm{~L}_{2}$ é variação de $\mathrm{PB}$ e inglês, esse comportamento só pode ser entendido em um "X potencial", denominado $\mathrm{C}$, então determinado pela equivalência e mudança. $\mathrm{C}$ é dado pela confluência entre equivalência e mudança na tradução (Quadro 3).

QUADRO 3 - Determinação de $\mathrm{PB} \cap$ inglês $=\mathrm{C}$ por Equivalência e Mudança.

\begin{tabular}{|c|c|c|}
\hline EQUIVALÊNCIA & MUDANÇA & TIPO DE RELAÇÃO \\
\hline$\checkmark$ & $\checkmark$ & PB $\cap$ ing $=\mathrm{C}$ \\
\hline$\checkmark$ & $\mathrm{x}$ & $-\cdots--$ \\
\hline $\mathrm{x}$ & $\checkmark$ & $-\cdots$ \\
\hline $\mathrm{x}$ & $\mathrm{x}$ & $\mathrm{PB} \cap$ ing $=\varnothing$ \\
\hline
\end{tabular}

Como é possível depreender da metodologia, a tradução é aqui empregada como ferramenta metodológica na investigação do contraste linguístico. Desta maneira, na próxima seção, serão utilizadas as relações de equivalência em tradução e correspondência formal, uma vez que o objetivo da seção é investigar a Interseção entre as línguas. Já na seção subsequente, serão utilizadas as relações de equivalência e mudanças na tradução, uma vez que o objetivo dessa última é investigar os recursos particulares de cada língua.

\section{Relações de equivalência e correspondência no ambiente multilíngue}

Seguindo os passos metodológicos para a análise dos dados, em primeiro lugar, foram analisados os recursos de cada língua separadamente (Quadro 4). 
QUADRO 4 - Recursos interpessoais (R) pertencentes a $\mathrm{L}_{1}$ ou $\mathrm{L}_{2}$.

\begin{tabular}{|c|c|c|c|c|c|}
\hline Nivel 1 & Nível 2 & Nível 3 & $\overline{\mathrm{PB}}$ & Inglês & Exemplos \\
\hline funções & funções & funções & $\left\{\mathrm{R} \in \mathrm{L}_{1}\right\}$ & $\left\{\mathrm{R} \in \mathrm{L}_{2}\right\}$ & \\
\hline \multirow[t]{4}{*}{ modo } & \multirow[t]{2}{*}{ indicativo } & declarativo & 50 & 41 & $\begin{array}{l}\text { - Já nos casamos. } \\
\text { - We are already married. }\end{array}$ \\
\hline & & interrog. & 15 & 16 & $\begin{array}{l}\text { - Como éque eu vou saber? } \\
\text { - How would I know? }\end{array}$ \\
\hline & \multirow[t]{2}{*}{ imperativo } & jussivo & 2 & 2 & $\begin{array}{l}\text { - Vá pra casa, Olivia. } \\
\text { - Go home, Olivia. }\end{array}$ \\
\hline & & sugestivo & --- & --- & --- \\
\hline \multirow[t]{7}{*}{ modalidade } & \multirow[t]{2}{*}{ modalização } & frequência & --- & --- & --- \\
\hline & & probabilid. & 1 & --- & - Não, claro que não. \\
\hline & \multirow[t]{2}{*}{ modulação } & obrigação & 9 & 8 & $\begin{array}{l}\text { - Bem, eu acho que terá de deixar. } \\
\text { - Wow, I think you have to. }\end{array}$ \\
\hline & & inclinação & --- & --- & --- \\
\hline & \multirow[t]{3}{*}{ grau } & baixo & --- & --- & --- \\
\hline & & médio & 7 & 7 & $\begin{array}{l}\text { - O Brick pode sair para brincar? } \\
\text { - Can Brick come out and play? }\end{array}$ \\
\hline & & alto & 3 & 1 & $\begin{array}{l}\text { - Bem, eu acho que terá de deixar. } \\
\text { - Wow, I think you have to. }\end{array}$ \\
\hline \multirow[t]{2}{*}{ polaridade } & positiva & & 47 & 45 & $\begin{array}{l}\text { - Já nos casamos. } \\
\text { - We are al ready married. }\end{array}$ \\
\hline & negativa & & 20 & 14 & $\begin{array}{l}\text { - Eu não sei nem do que que eu tô sendo } \\
\text { acusado. } \\
\text { - I don't even know what I'm being accused of. }\end{array}$ \\
\hline \multirow{4}{*}{$\begin{array}{l}\text { avaliação } \\
\text { modal }\end{array}$} & \multirow[t]{2}{*}{ persuasão } & obediência & --- & --- & --- \\
\hline & & resposta & --- & --- & --- \\
\hline & \multirow[t]{2}{*}{ verificação } & prosódia & 1 & --- & - Ué, as pessoas fazem coisas loucas, né? \\
\hline & & papel & 6 & --- & - Ué, as pessoas fazem coisas loucas, né? \\
\hline
\end{tabular}

A Disjunção - isto é, quando as línguas $\mathrm{L}_{1}$ e $\mathrm{L}_{2}$ não possuem recursos comuns, e com isso, são completamente diferentes, $\mathrm{L}_{1} \cap \mathrm{L}_{2}=\varnothing$ - foi o primeiro parâmetro adotado. Conforme os recursos encontrados no ambiente multilíngue desta pesquisa, $\mathrm{L}_{1}$ e $\mathrm{L}_{2}$ são descritos da seguinte forma.

$\mathrm{L}_{1}$ apresenta MODO, MODALIDADE, POLARIDADE e AVALIAÇÃO. O MODO possui todos os recursos do Indicativo, porém, o Imperativo tem apenas um nível de delicadeza, na opção Jussivo. Para a MODALIDADE, a Modalização apresenta a opção de Probabilidade e a modulação a Obrigação. O Grau é formado por duas opções, Médio e Alto. A POLARIDADE possui Positiva e Negativa. Já a AVALIAÇÃO MODAL, apenas as opções mais delicadas da Verificação. O contraste entre o "PB em potencial" e $\mathrm{L}_{1}$ pode ser visto na Figura 2. 


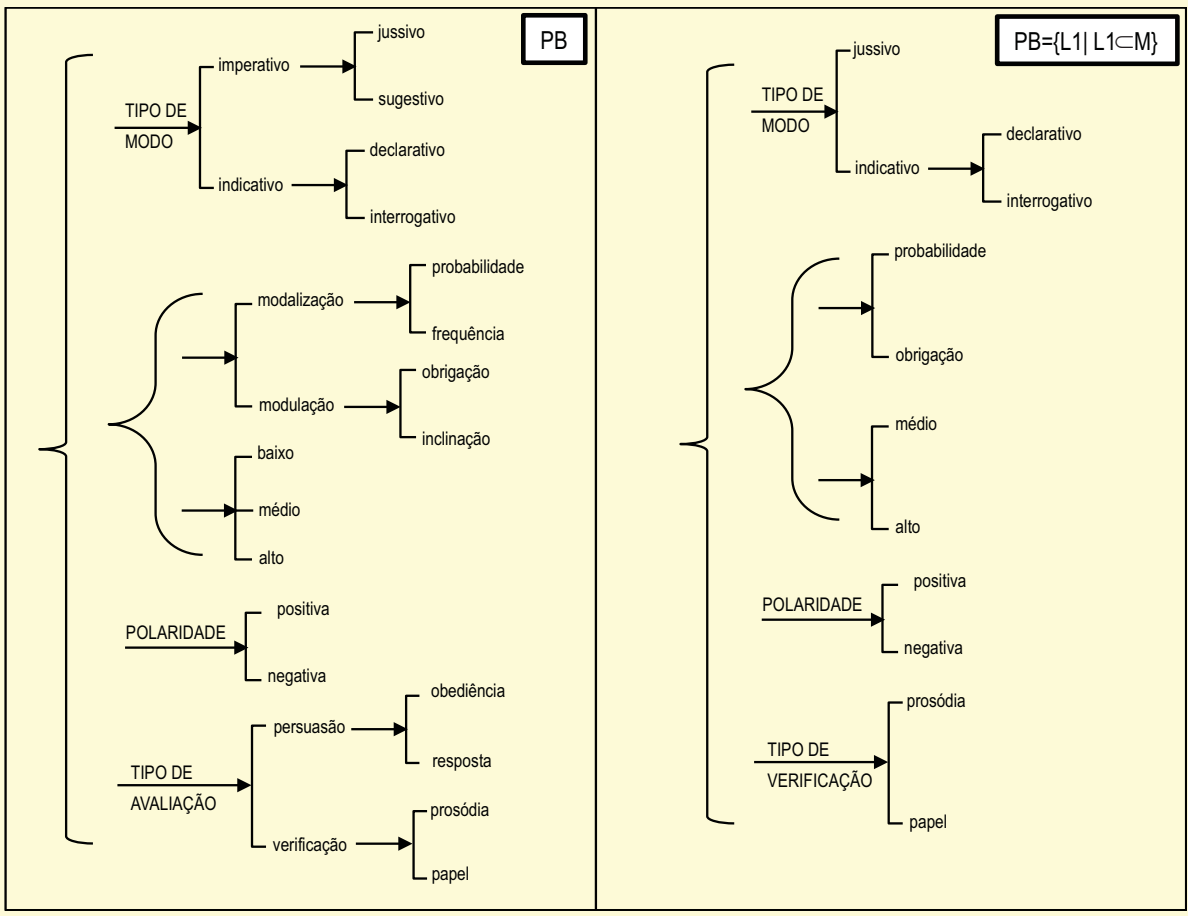

FIGURA 2 - Contraste entre PB e PB $=\left\{\mathrm{L}_{1} \mid \mathrm{L}_{1} \subset \mathrm{M}\right\}$

Para os sistemas de $\mathrm{L}_{2}$, o MODO apresenta Indicativo e apenas o Jussivo para o Imperativo. A MODALIDADE se resume à Obrigação. O Grau se divide em Médio e Alto. A POLARIDADE é Positiva e Negativa. O contraste entre $\mathrm{L}_{2}$ e o "inglês em potencial" se apresenta na Figura 3. 


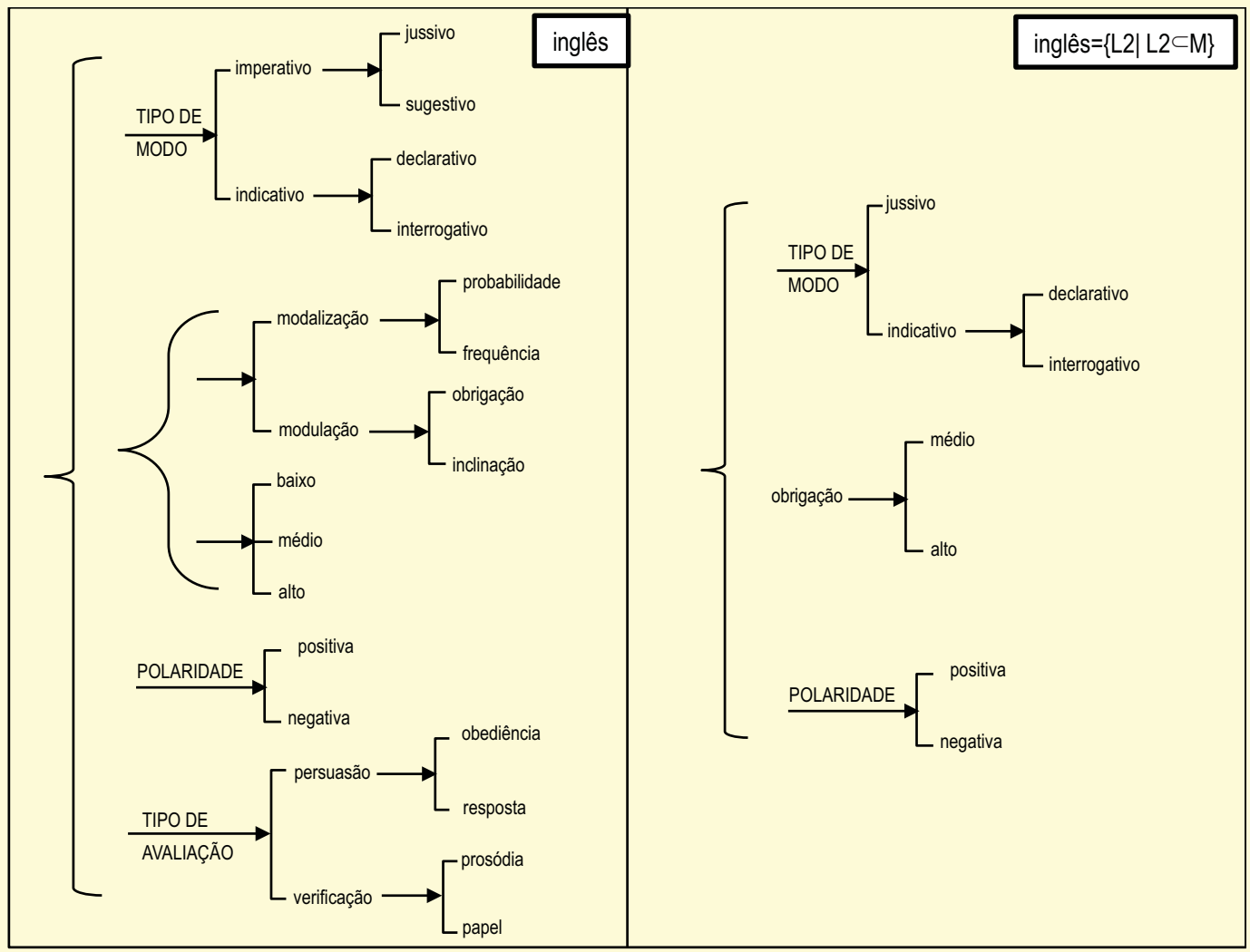

FIGURA 3 - Contraste entre inglês e inglês $=\left\{\mathrm{L}_{2} \mid \mathrm{L}_{2} \subset \mathrm{M}\right\}$

A partir da Disjunção, tem-se os subconjuntos $\mathrm{L}_{1}$ e $\mathrm{L}_{2}$. $\mathrm{L}_{1}=\{$ Declarativo, Interrogativo, Jussivo, Probabilidade, Obrigação, Médio, Alto, Positiva, Negativa, Prosódia, Papel-Falante\}. $\mathrm{L}_{2}=\{$ Declarativo, Interrogativo, Jussivo, Obrigação, Médio, Alto, Positiva, Negativa, Papel-Falante\}. Logo, M é definido como o conjunto de todos os recurso presentes no ambiente multilíngue, ou $\mathrm{M}=\left\{\mathrm{L}_{1} \cup \mathrm{L}_{2}\right\}=\left\{\mathrm{R} \mid \mathrm{R} \in \mathrm{L}_{1} \vee \mathrm{R} \in \mathrm{L}_{2}\right\}=\{$ Declarativo, Interrogativo, Jussivo, Probabilidade, Obrigação, Médio, Alto, Positiva, Negativa, Prosódia, Papel-Falante\}.

Ressalta-se que, sob um primeiro olhar, os recursos de $\mathrm{L}_{2}$ estariam todos contidos em $\mathrm{L}_{1}$, ou $\mathrm{L}_{2} \subset \mathrm{L}_{1}$. Contudo, isso só é possível de ser observado quando as línguas são comparadas. Se $\mathrm{L}_{1}$ e $\mathrm{L}_{2}$ 
forem tomadas isoladamente, uma dada função de $\mathrm{L}_{1}$ (ou de $\mathrm{L}_{2}$ ) não se compara a quaisquer funções de outras línguas (cf. HALLIDAY, 2002). Esse tipo de constatação se baseia apenas na descrição geral das funções para a língua como um todo. Ela ainda não leva em conta o papel na organização interna da gramática - ou a reacomodação dos sistemas para um ambiente multilíngue determinado. Assim, é a existência do ambiente multilíngue que permite a comparação.

Por esse motivo, a comparação entre $\mathrm{L}_{1}$ e $\mathrm{L}_{2}$ não é feita pelo simples cotejo entre categorias. A comparação se faz pela Interseção entre $\mathrm{L}_{1}$ e $\mathrm{L}_{2}$ - isto é, quando as línguas $\mathrm{L}_{1}$ e $\mathrm{L}_{2}$ compartilham um conjunto comum de recursos (denominado $\mathrm{X}$ ). Tendo a tradução como ferramenta para a identificação dos recursos compartilhados pelas línguas, por meio da Interseção, determinou-se $\mathrm{X}$ (Quadro 5):

QUADRO 5 - Equivalência e Correspondência na determinação de $\mathrm{L}_{1} \cap \mathrm{L}_{2}=\mathrm{X}$ da pesquisa.

\begin{tabular}{|l|l|l|}
\hline EQUIVALÊNCIA & CORRESPONDÊNCIA & $\mathrm{N}^{\circ}$ DE OCORRÊNCIAS \\
\hline equivalente & correspondente & $\mathbf{4 9}$ \\
\hline equivalente & não & $\mathbf{1 1}$ \\
\hline não & correspondente & $\mathbf{0}$ \\
\hline não & não & $\mathbf{5}$ \\
\hline
\end{tabular}

Com a determinação de $\mathrm{X}$, é então possível apresentar a constituição $\mathrm{M}$ a partir dos subconjuntos relevantes para esta pesquisa (Figura 4). 


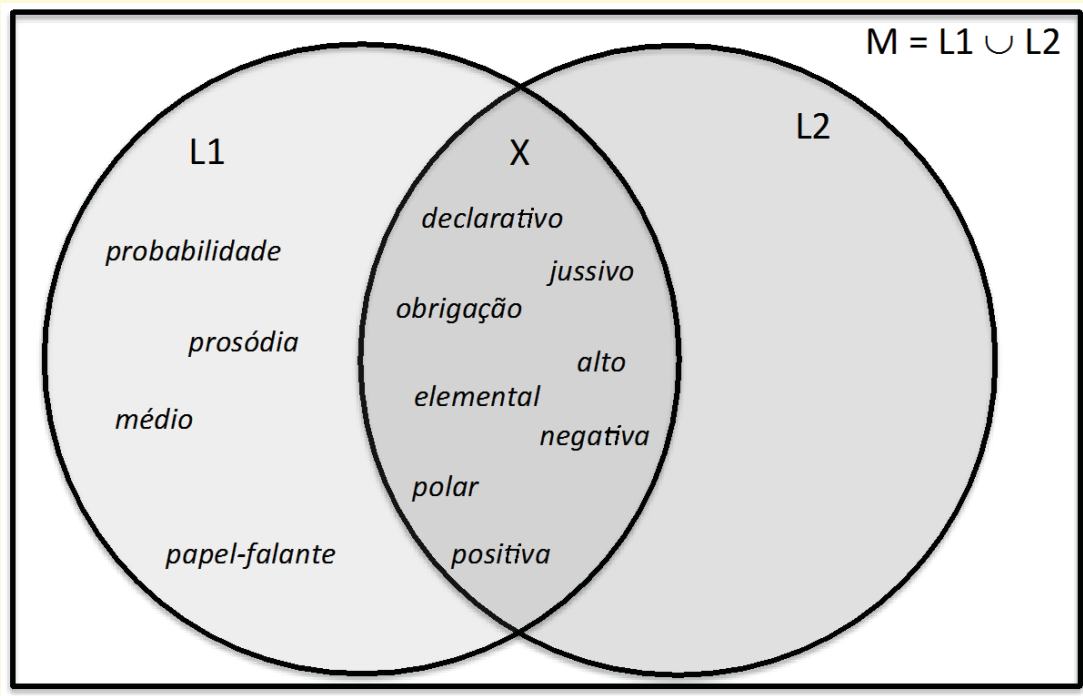

FIGURA 4 - Interseção entre $\mathrm{L}_{1}$ e $\mathrm{L}_{2}$.

A Figura 4 mostra como se distribuem os recursos em M. Através dela, nota-se que as línguas $\mathrm{L}_{1}$ e $\mathrm{L}_{2}$ compartilham Declarativo, Jussivo, Obrigação, Alto, Elemental, Polar, Negativa e Positiva ${ }^{3}$ Logo, em $\mathrm{M}$, esses não são recursos de $\mathrm{L}_{1}$ ou $\mathrm{L}_{2}$, mas de ambas as línguas.

Extrapolando o modelo para a língua, funções como Declarativo, Jussivo e Obrigação são recursos compartilhados pelo PB e pelo inglês, estando assim presentes nas duas línguas. Pelo mesmo princípio, Probabilidade, Prosódia, Médio e Papel-Falante ${ }^{4}$ são recursos exclusivos de $\mathrm{L}_{1}$ em $\mathrm{M}$. Extrapolando $\mathrm{M}$ para a língua, funções como Probabilidade e Prosódia são particulares do PB e não estão presentes na língua inglesa.

Esse, então, é o modelo do ambiente multilíngue para o corpus da presente pesquisa. A partir dele é possível observar quais as contribuições de cada sistema linguístico para a constituição dos corpora, bem como quais recursos são comuns às línguas - i.e., multilíngues. Com isso, o desenvolvimento deste modelo pode contribuir com as abordagens sistêmicas da tradução, na sua vertente multilíngue, das seguintes formas: 
(i) Em primeiro lugar, oferece um modelo com base nos aspectos sistêmicos da tradução, e não apenas através das categorias funcionais da gramática. (ii) Além disso, evita o problema de uma das línguas ser tomada como original, o que levaria a análise a utilizar essa língua como parâmetro de comparação para as línguas traduzidas - isto é, as semelhanças e diferenças entre os textos original e traduzido teriam, necessariamente, como base o original. (iii) Por conseguinte, é possível prescindir do tertium comparationis externo às línguas e às categorias de análise, uma vez que as relações de Disjunção e Interseção, bem como as relações de equivalência, correspondência e mudança são suficientes para estabelecer a comparação entre as línguas. (iv) $\mathrm{O}$ modelo pode ser extrapolado para a língua, prevendo, assim, categorias específicas de cada uma delas para pesquisas futuras. (v) Por fim, o escopo das pesquisas afiliadas às abordagens sistêmicas da tradução se ampliam, uma vez que a tradução pode ser utilizada como ferramenta de análise para outros fenômenos do contato linguístico, tais como o contraste entre as línguas e a produção multilíngue.

Contudo, esse ambiente multilíngue aponta para novos questionamentos. Por exemplo, sabe-se que a Probabilidade é um recurso da língua inglesa (cf. HALLIDAY e MATTHIESSEN, 2014), pois está presente na descrição que embasou a presente análise.

Cabe, portanto, perguntar se a ausência de recursos como, por exemplo, a Probabilidade em $\mathrm{M}$ é derivada do contato linguístico. Além disso, uma vez que apenas $\mathrm{L}_{1}$ apresenta recursos exclusivos em M, mas no corpus de M existem textos comparáveis e paralelos nas duas direções, cabe perguntar por que $\mathrm{L}_{2}$ não apresenta recursos exclusivos em $\mathrm{M}$. Ou ainda, em que medida $\mathrm{L}_{1}$ se comporta como o $\mathrm{PB}$, e $\mathrm{L}_{2}$ se comporta como o inglês.

Para investigar essas perguntas, é necessário dar mais um passo na modelagem e tomar o comportamento gramatical como sendo uma variação em $\mathrm{M}$ das línguas $\mathrm{PB}$ e inglês. 


\section{Comportamento gramatical multilíngue: contribuições para o estudo da tradução e do contraste linguístico}

O propósito da descrição sistêmica é apresentar o potencial de uso da língua, não sendo seu papel principal explicar como os recursos são efetivamente utilizados (MATTHIESSEN et al., 2010). O sistema linguístico "em uso", de outra forma, pode ser explicado pela dinâmica discursiva (LEMKE, 1993).

A dinâmica gramatical, por sua vez, revela como são utilizados os recursos gramaticais. O termo comportamento gramatical designa cada uma das maneiras pelas quais a dinâmica gramatical acontece. Aqui, a noção de 'comportamento' implica em variação, e o comportamento de um determinado recurso gramatical (ou a 'dinâmica gramatical de um recurso') sugere o tanto que o uso desse recurso varia conforme a modelagem do ambiente do qual é parte. Consequentemente, a modelagem estabelece a base para se investigarem os fenômenos relativos ao comportamento gramatical multilíngue; e as relações de equivalência em tradução e correspondência tornam-se, então, ferramentas dessa investigação.

$\mathrm{O}$ ambiente multilíngue do presente trabalho se configura como o conjunto universo para a modelagem dos recursos gramaticais interpessoais da oração. Aqui, o emprego das relações de equivalência em tradução e correspondência determinam quais recursos são comuns às línguas, ou distintos para cada uma delas, possibilitando modelar os subconjuntos $\mathrm{L}_{1}, \mathrm{~L}_{2}$ e X em $\mathrm{M}$.

Contudo, essa modelagem não é suficiente, por si só, para explicar o contato linguístico, devendo ser complementada. Por um lado, se por meio das relações de equivalência em tradução e correspondência, determinou-se que $\mathrm{L}_{2} \subset \mathrm{L}_{1}$ e $\mathrm{L}_{1}=\mathrm{M}$, por outro lado, são as relações de equivalência e mudança em tradução que explicam o comportamento gramatical multilíngue.

A comparação entre $L_{1}$ e $L_{2}$ passa a ser feita em razão das possibilidades que as línguas PB e inglês oferecem, mas que não foram empregadas. Assim, a Interseção a ser determinada seria um conjunto potencial C (Quadro 6): 
QUADRO 6 - Equivalência e Mudança na determinação de $\mathrm{PB} \cap$ ing $=\mathrm{C}$.

\begin{tabular}{|l|l|l|}
\hline EQUIVALÊNCIA & MUDANÇA & $\mathrm{N}^{\circ}$ DE OCORRÊNCIAS \\
\hline equivalente & correspondente & $\mathbf{4 9}$ \\
\hline equivalente & mudança & $\mathbf{1 1}$ \\
\hline não & correspondente & $\mathbf{0}$ \\
\hline não & não & $\mathbf{5}$ \\
\hline
\end{tabular}

Com a determinação de $\mathrm{X}$ e $\mathrm{C}$, é então possível apresentar a constituição $M$ tanto a partir dos subconjuntos $L_{1}$ e $L_{2}$ como das línguas PB e inglês (Figura 5).

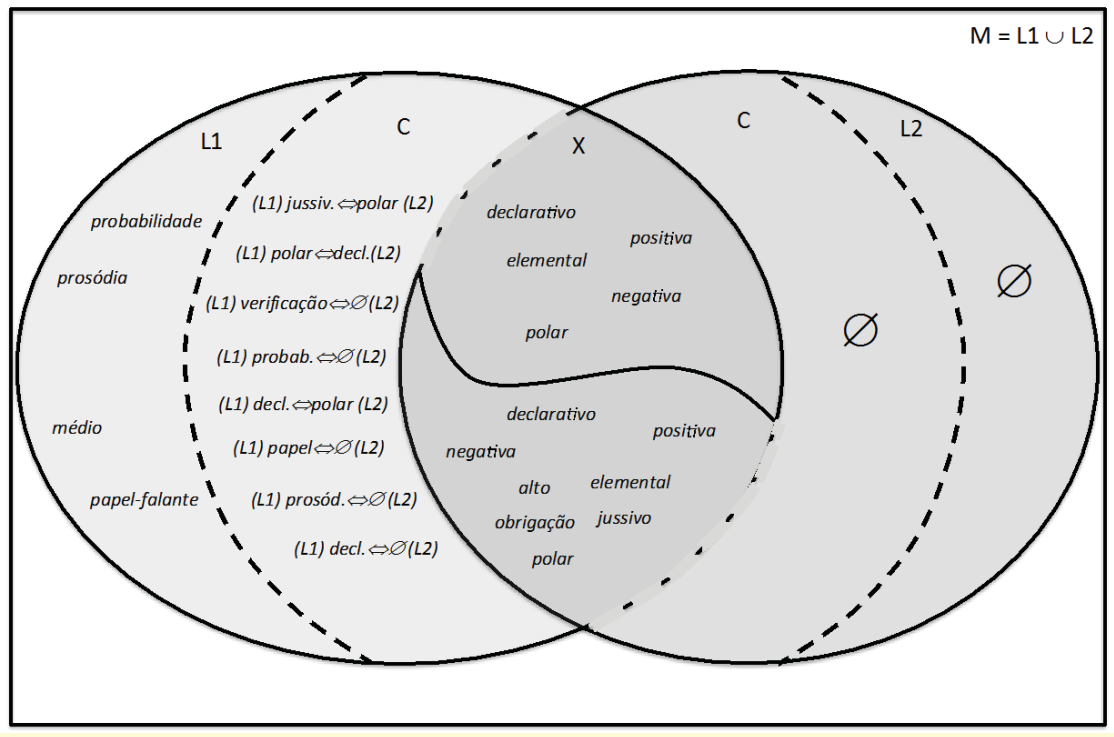

FIGURA 5 - Equivalência e mudança no ambiente multilíngue.

A Figura 5 mostra como, considerando a mudança, se distribuem os recursos em M. Através dela, nota-se que os recursos de $\mathrm{L}_{1}$ foram sempre traduzidos por recursos de $\mathrm{X}$, tais como $\mathrm{L}_{1}$ 
(jussivo) $\rightarrow \mathrm{L}_{2}$ (polar), ou $\mathrm{L}_{1}$ (declarativo) $\rightarrow \mathrm{L}_{2}$ (polar). Nenhum recurso exclusivo de $\mathrm{L}_{2}$ foi empregado (Quadro 7); daí $\mathrm{L}_{2}-\mathrm{L}_{1}=\varnothing$.

QUADRO 7 - Exemplos de equivalência e mudança no corpus.

\begin{tabular}{|l|l|l|}
\hline mudança & $\mathrm{L}_{1}$ & $\mathrm{~L}_{2}$ \\
\hline$\left(\mathrm{L}_{1}\right)$ papel $\Leftrightarrow \varnothing\left(\mathrm{L}_{2}\right)$ & $\begin{array}{l}\text { Ah, sei lá, Vani. } \\
\text { Como é que eu vou } \\
\text { saber? }\end{array}$ & How would I know? \\
\hline$\left(\mathrm{L}_{1}\right)$ jussi v. $\Leftrightarrow$ polar $\left(\mathrm{L}_{2}\right)$ & $\begin{array}{l}\text { Veja bem o nível de } \\
\text { paranóia... }\end{array}$ & $\begin{array}{l}\text { You see the level of } \\
\text { paranoia? }\end{array}$ \\
\hline$\left(\mathrm{L}_{1}\right)$ decl. $\Leftrightarrow$ polar $\left(\mathrm{L}_{2}\right)$ & $\begin{array}{l}\text { Cê trouxe aquela mu- } \\
\text { lher aqui, Sérgio? }\end{array}$ & $\begin{array}{l}\text { You brought that girl } \\
\text { in here! }\end{array}$ \\
\hline$\left(\mathrm{L}_{1}\right)$ prosódia $\Leftrightarrow \varnothing\left(\mathrm{L}_{2}\right)$ & $\begin{array}{l}\text { Ah, não troexe no } \\
\text { seu quarto, ela ficou } \\
\text { o tempo todo na sala. }\end{array}$ & $\begin{array}{l}\text { Was she in the living } \\
\text { room the whole time? }\end{array}$ \\
\hline$\left(\mathrm{L}_{1}\right)$ verificação $\Leftrightarrow \varnothing\left(\mathrm{L}_{2}\right)$ & $\begin{array}{l}\text { Ué, as pessoas fazem } \\
\text { coisas loucas, né? }\end{array}$ & People do crazy stuff. \\
\hline
\end{tabular}

Por u m lado $\mathrm{L}_{2}$ poderia ser considerada "mais multilíngue" que $\mathrm{L}_{1}$, uma vez que $\mathrm{L}_{2}=\mathrm{X}$. Contudo, por outro lado, $\mathrm{L}_{1}$ poderia também ser considerada "mais multilíngue" porque utiliza recursos de $\mathrm{L}_{1}$, de $\mathrm{X}$ e, consequentemente de $\mathrm{L}_{2}$ - em outras palavras, emprega todos os recursos de $\mathrm{M}$.

O conceito 'multilíngue', logo, é utilizado tanto para se referir ao grau de Interseção entre subconjuntos (como no caso de $\mathrm{L}_{2}$ ), quanto à variedade de recursos de um subconjunto (como no caso de $\mathrm{L}_{1}$ ). No caso de $\mathrm{L}_{2}$, "mais multilíngue" implica em "mais pervasivo"; no caso de $\mathrm{L}_{1}$, implica em "mais diversificado". 


\section{Conclusão}

Tendo como objetivo empregar a equivalência tradutória (CATFORD, 1965; MATTHIESSEN, 2001) como metodologia para a investigação do ambiente multilíngue, este artigo partiu das Abordagens Sistêmicas da Tradução (PAGANO e VASCONCELLOS, 2005), na sua interface com os Estudos Multilíngues (MATTHIESSEN et al., 2008), para propor um modelo do ambiente multilíngue como um conjunto de recursos gramaticais.

Por conta do caráter insipiente, os Estudos Multilíngues carecem da consolidação de uma metodologia eficiente na modelagem do ambiente multilíngue. Por esse motivo, o presente artigo propôs empregar as relações de equivalência como método para o estudo de outros tipos de relação entre línguas, focando-se especificamente no contraste.

Embasado em Halliday (2001), o qual aponta que não é possível determinar categorias universais para todas as línguas, o estabelecimento das relações de equivalência tradutória como ferramenta da modelagem propiciou a seguinte hipótese de trabalho: modelar o ambiente multilíngue como um conjunto de todos os recursos de todas as línguas que o constituem. O modelo então prevê que o ambiente multilíngue é constituído pela produção de significado, é supra-línguas, e a separação entre as línguas se dá no limite entre sua Interseção e Disjunção; todas as línguas pertencem à mesma classe, uma vez que são subconjuntos; o comportamento gramatical multilíngue é uma variação do comportamento da língua.

No curso da pesquisa, o ambiente multilíngue modelado concentrou-se nos recursos gramaticais interpessoais da oração. Ele conseguiu estabelecer quais recursos são comuns às línguas, ou distintos a cada uma delas, bem como descreveu os subconjuntos relativos tanto às línguas quanto aos recursos comuns a elas. $\mathrm{O}$ comportamento gramatical do contato linguístico, por sua vez, foi descrito como uma variação do uso da língua porque só pode ser investigado quando o ambiente multilíngue é comparado com a língua como um todo, tal como previra o modelo. 
Em seguida, apontou-se como a tradução pode ser empregada como ferramenta de comparação das línguas, uma vez que evita a comparação das línguas como um todo, concentrando-se na variação gerada em cada ambiente multilíngue - ou o papel que cada língua exerce na criação dos significados de um só texto.

No que tange às contribuições para a análise contrastiva, a tradução permite que se amplie para além da correspondência formal generalizada das línguas como um todo, podendo se basear nos tipos de contraste de um ambiente multilíngue específico. Como exemplo, citam-se os dados do presente artigo, em que a função jussivo é contraste de polar; polar de declarativo; e a verificação e a probabilidade não são comparáveis.

Além disso, destaca-se que as relações comumente designadas como 'multilíngue' abrangem tanto o grau de Interseção entre subconjuntos (como no caso de $\mathrm{L}_{2}$ ), quanto à variedade de recursos de um subconjunto (como no caso de $\mathrm{L}_{1}$ ). Isso implica na necessidade de se acrescentarem esses dois conceitos - pervasão e diversificação - ao comportamento gramatical, para se entender como ele acontece no ambiente multilíngue.

Este artigo espera, por fim, poder contribuir com a ampliação do papel das Abordagens Sistêmicas da Tradução, no que tange aos aspectos sistêmicos da produção de significado por mais de uma língua. No cenário das Abordagens Sistêmicas da Tradução, foi possível estabelecer um diálogo produtivo entre os Estudos da Tradução e os Estudos Multilíngues. Por um lado, os Estudos da Tradução ampliam seu potencial de atuação, uma vez que, na condição de um tipo especializado do contato linguístico, operam como ferramenta metodológica para o estudo de outros tipos de contato. Os Estudos da Tradução poderão, assim, ocupar o espaço metodológico de pesquisas, por exemplo, em linguística contrastiva e ensino de língua estrangeira. Por outro lado, os Estudos Multilíngues, que ainda não possuem um corpo metodológico sólido para lidar com a multiplicidade de fenômenos do contato linguístico, se beneficiam dos Estudos da Tradução pois encontram nas relações geradas pelo fenômeno tradutório - equivalência, correspondência e mudança 
em tradução - uma forma segura de comparação entre as línguas, bem como um conjunto de critérios para apontar a contribuição efetiva de cada língua ao ambiente multilíngue.

\section{Notas}

1. The description of a language... may be referred to forms of the language itself, or to forms of ... other languages or to non-formal-linguistic concepts... There can be no universal formal-linguistic categories (there might theoretically be categories formally identified as common to all languages studied here-to-fore, but such identification is not yet a practical possibility), while non-formal-linguistic categories, if they are to figure in the description at all, must be implicitly regarded as universal”.

2. A formalidade da notação sobre conjuntos foi retirada da teoria de conjuntos; contudo, a nomenclatura $\mathrm{M}, \mathrm{L}, \mathrm{R}$, etc. para os subconjuntos do ambiente multilíngue são apresentadas por primeira vez no presente artigo.

3. Para um maior aprofundamento acerca destas categorias da gramática interpessoal, favor conferir Halliday e Matthiessen (2013) para o inglês; e Figueredo (2011) para o PB.

4. Cf. Halliday e Matthiessen (2013) para o inglês; e Figueredo (2011) para o PB. 


\section{Referências}

CATFORD, J. A linguistic theory of translation: an essay in applied linguistics. London: Oxford Univ., 1965. 103p.

FIGUEREDO, G. Introdução ao perfil metafuncional do português brasileiro: contribuições para os estudos multilíngues. (2011). 385 p. Tese - Programa de Pós-Graduação em Estudos Linguísticos, Faculdade de Letras, Universidade Federal de Minas Gerais, Belo Horizonte, 2011.

GLEASON, H. 1965. Linguistics and English grammar. New York: Holt, Rinehart \& Winston. HALLIDAY, M. On grammar. London: Continuum, 2002.

HALLIDAY, M. Towards the theory of a good translation. In: STEINER, E.; YALLOP, C (Ed.). Exploring translation and multilingual text production: beyond content. Berlin/ New York: Mouton de Gruyter, 2001. p. 13-18.

HALLIDAY, M.; MATTHIESSEN, C. Introduction to functional grammar (4a Ed.). London: Routledge, 2013.

KENNING, M. M. What are parallel and comparable corpora and how can we use them? In: KEENE, A.; MCCARTHY, M. (Ed.) The Routledge handbook of corpus linguistics. Madison: Routledge, 2010. p. 487-500.

LEMKE, J. Discourse, Dynamics, and Social Change. Cultural Dynamics 6(1): 243-275, 1993.

MARTIN, J. English text: system and structure. Philadelphia and Amsterdam: John Benjamins, 1992.

MARTIN, J. Systemic functional grammar: a next step into the theory - axial relations. Beijing: Higher Education Press, 2013. MATTHIESSEN, C. TERUYA, K.; LAM, M. Key terms in systemic functional linguistics. London e New York: Continuum, 2010. 
MATTHIESSEN, C. The environments of translation. In: STEINER, E. YALLOP, C. (Eds.). Exploring translation and multilingual text production: beyond content. Berlin \& New York: Mouton de Gruyer, 2001. p. 41-124.

MATTHIESSEN, C.; TERUYA, K.; WU, C. Multilingual studies as a multi-dimensional space of interconnected language studies. In: WEBSTER, J. (Ed.). Meaning in Context: implementing intelligent applications of language studies. London and New York: Continuum, 2008.

PAGANO, A.; VASCONCELLOS, M. L. Explorando interfaces: estudos da tradução, linguística sistêmico-funcional e linguística de córpus. In: ALVES, F., MAGAlHÃES, C., PAGANO, A. (Org.). Competência em tradução: cognição e discurso. Belo Horizonte: Editora da UFMG, 2005. p. 177-207.

STEINER, E.; YALLOP, C (Ed.). Exploring translation and multilingual text production: beyond content. Berlin/ New York: Mouton de Gruyter, 2001.

TEICH, E. Contrastive linguistics and translation studies revisited. In: GIL, Alberto (Org.) Modelle der Translation: Grundlagen fur Methodik, Bewertung, Computermodellierung. Frankfurt am Main \& Berlin: Lang, 1999.

TEICH, E. Cross-linguistic variation in system and text: a methodology for the investigation of translations and comparable texts. Berlin and New York: Mouton de Gruyter, 2003.

Recebido em: 12/01/2015

Aceito em: 03/02/2015 